N. P. Medvedeva ${ }^{1}$, I. S. Sapozhnikova ${ }^{1}$, V. L. Rusinovi, ${ }^{1,}$ E. N. Ulomskii ${ }^{1}$

${ }^{1}$ Ural Federal University, 19 Mira Street, 620002, Ekaterinburg, Russia. E-mail: nataly-medvedeva@yandex.ru ${ }^{2}$ Institute of Organic Synthesis Ural Division of RAS, 22 Kovalevskaya Street/20 Academicheskaya Street, 620990, Ekaterinburg, Russia.

E-mail:v.l.rusinov@urfu.ru

\title{
The redox transformations and nucleophilic replacements as possible metabolic reactions of the drug "Triazaverin". The chemical modeling of the metabolic processes
}

As a model of metabolic transformations of antiviral drug "Triazaverin" and its analogues-2-alkylthio-6-nitro-1,2,4-triazolo[5,1-c][1,2,4]triazine-7-ones la-d examined the oxidation of alkylthio groups to the corresponding sulfoxides $2 a-d$ and sulfones $3 a-d$, as well as the process of nucleophilic substitution sulfonyloxy group of cysteine and cysteamine with the formation of compounds 5 and 6.

Key words: biological active compounds; heterocycles; triazine; Triazaverin; antiviral drug.

(c) Medvedeva N. P., Sapozhnikova I. S., Rusinov V. L., Ulomskii E. N., 2015

\section{Introduction}

The relevance of creating new antiviral drugs due to the joint action of such operating factors as the spread of socially significant, particularly dangerous infections, and the emergence of pathogenic viral strains resistant to existing drugs.

The antiviral drug "Triazaverin" and its analogues are highly effective in experiments in vivo and decreased activity in experiments on cell cultures, suggesting that the antiviral effect is not the "Triazaverin", and products of its transformations in the body. One way to identify

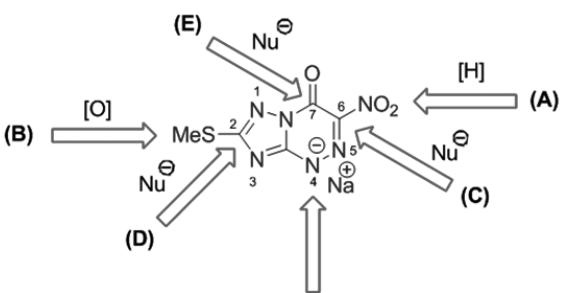

(F)

such transformations in the organism is to predict the possible products of modifications of the compounds and chemical synthesis models.

Based on the molecular structure of compounds 1a and study of chemi- 
cal properties of nitroazolo[5,1-c][1,2,4] triazines [1] can assume various variants of metabolism: redox transformations in the body (directions A, B) as the reduction of the nitro group under the action of a reductases (direction $\mathrm{A}$ ), oxidation of alkylthio fragment under the action of oxidases (direction B) and its further transformation. The part transferases under the action of $\mathrm{N}-$ and S-nucleophils, such as lysine, arginine, cysteine could lead to the replacement of alkylthio- or nitro groups (directions $\mathrm{C}, \mathrm{D}$ ).

The hydrolytic enzymes are also quite capable to lead the transformation of "Tri- azaverin" accompanying the degradation of triazine cycle with the break of bond $\mathrm{C}-\mathrm{N}$ (direction E). The alkylation on the $\mathrm{N}$-atom is possible too (direction $\mathrm{F}$ ).

With regard to the redox transformations of "Triazaverin" that we previously established that after the intragastric administration of laboratory animals of the drug substance, there is a reduction of the nitro group with the formation of 2-methylthio-6-amino-1,2,4-triazolo[5,1c] $[1,2,4]$ triazine-7-one [2], does not exhibit antiviral action in vitro and, most likely, which is not applicable metabolite.

\section{Results and Discussion}

Considering the possibility of oxidation of alkylthio groups under use "Triazaverin" and its analogues, it should be noted that in the majority of viral infections at an early stage of the process is increased generation of reactive oxygen species [3]. Based on the foregoing, it is natural to assume that in these conditions can occur in the oxidation reaction of S-methyl group of "Triazaverin" characteristic, for example, for SH-containing amino acids of methionine under oxidative stress.

In the present communication present data on the production model experiments with the drug "Triazaverin" - the study of the oxidation of S-methyl group and the behavior of oxidation products under the action of S-nucleophils. This approach allows, on the one hand, to pre-

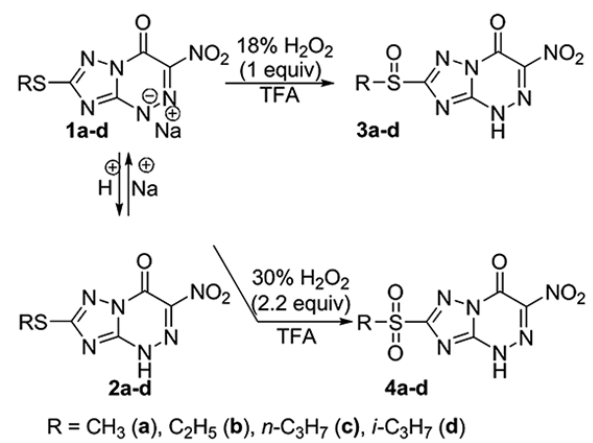

Scheme 1

dict the course of chemical reactions related to the behavior of the drug in the body, and on the other hand, by the synthesis of the respective compounds to simulate the process of formation of covalent bonds of azolo[5,1-c]1,2,4-triazines with S-key fragments of proteins as cells and virus.

\section{Redox-transformations of "Triazaverin" and its derivatives}

The first aspect of this work was the synthesis of model compounds - oxidation of sulfhydryl groups in molecules of sodium salts of 2-alkylthio-6-nitro-1,2,4- triazolo[5,1-c]1,2,4-triazine-7-ones $1 \mathrm{a}$-d or in the associated N-H-acids comprising the formation of heterocyclic sulfoxides 3a-d and sulfones $4 a-d$. Sulfoxides 
3a-d (Scheme 1) were obtained under the treatment of the compounds 1a-d or 2a-d by the equimolar quantity of $18 \%$ hydrogen peroxide in the trifluoroacetic acid.

Further oxidation to the corresponding sulfones 4a-d with the yields $62-71 \%$ carried out by gradual addition of excess 2.2 equivalent of $30 \%$ hydrogen peroxide to the suspension of 2-alkylthio-1,2,4triazolo[5,1-c]-triazines in the trifluoroacetic acid at room temperature.

The represented reactions model the possible metabolic transformations of "Triazaverin" and its derivatives under the action of the active forms of oxygen including hydrogen peroxide.

Nucleophilic replacement of methylsulfonilic group. It is known that alkylsulfonyl fragments are susceptible to the substitution reactions when interacting with nucleophiles [4]. With regard to the behavior of nitro group in "Triazaverin" but the susceptibility of the nitro group associated with aromatic (heteroaromatic) cycle to the displacement under the nucleophiles [5] is well known.

Thus in the structure of 2-alkylsulfonyl-1,2,4-triazolo[5,1-c]1,2,4-triazine-7-ones $4 \mathrm{a}-\mathbf{d}$ there are two groups that are susceptible to nucleophilic displacement. This situation is of interest for the research of the comparative reactive ability of the easy outgoing groups $\mathrm{CH}_{3} \mathrm{SO}_{2}$ in 1,2,4-triazole and $\mathrm{NO}_{2}$ in 1,2,4-triazine cycles in the compounds $4 \mathrm{a}-\mathrm{d}$ and also as the model of the behavior of "Triazaverin" in the organism. As the nucleophilic reagents in this work were used S-nucleophiles(cysteine and cysteamine), which can be regarded as models of protein fragments containing cysteine fragment.

The substitution sulfonylic fragment in 2-methylsulfonyl-1,2,4-triazolo[5,1c]1,2,4-triazine $4 \mathrm{a}$ under the influence, as cysteine and cysteamine, occurs in dry methanol in the presence of triethylamine in a few days with the formation of compounds 5 and 6- substitution products methylsulfonyl group with the yields 4146 \% (Scheme 2).

The date of NMR ${ }^{1} \mathrm{H}$, IR-spectroscopy and elemental analysis for the compounds 5, 6 correspond to the attributed structures.

The described displacement of alkylsulfonyl group in the compound $4 \mathrm{a}$ is the argument in favor of the assumption about the possible participation of this fragment in the metabolic transformations of "Triazaverin".

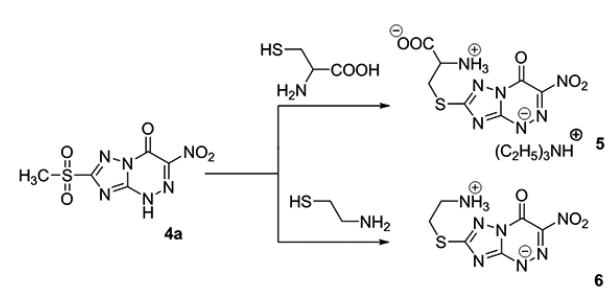

Scheme 2

\section{Experiment part}

$\mathrm{NMR}^{1} \mathrm{H}$ and ${ }^{13} \mathrm{C}$ spectra were recorded on a spectrometer Bruker DRX-400 (400 and $100 \mathrm{MHz}$, respectively) in DMSO- $\mathrm{d}_{6}$ and $\mathrm{D}_{2} \mathrm{O}$. The chemical shifts are recorded in $\sigma$ scale relatively internal standard TMS for $\mathrm{NMR}^{1} \mathrm{H}$ spectra.

Elemental analyses were performed on CHNS-analyzer "Perkin Elmer 2400-II". IR spectra $\left(4000-400 \mathrm{~cm}^{-1}\right)$ of the received compounds were recorded on a spectrophotometer "Perkin Elmer Spectrum One BFTIR" in thin layer of a 
sample (DRA). Reaction monitoring and the individuality of the synthesized compounds was performed by TLC plates Sorbfil in the systems: ethyl acetate and butanol-acetic acid-water 4:1:1.

General methods 1 for preparation of 2-alkylsulfinyl-6-nitro-1,2,4-triazolo[5,1c]1,2,4-triazin-7-ones (3a-d).

To a suspension $0.01 \mathrm{~mol}$ of sodium salt 2-alkylthio-6-nitro-1,2,4triazolo[5,1-c]1,2,4-triazin-7-one (1) in $10 \mathrm{~mL}$ of trifluoroacetic acid by mixing was added $1.78 \mathrm{ml}$ ( 1 eq.) of $18 \%$ hydrogen peroxide. The reaction mass is mixed at room temperature during 3 hours. The resulting solid precipitate was filtered and was crystallized from iso-propanol.

2-Methylsulfinyl-6-nitro-1,2,4triazolo[5,1-c]1,2,4-triazine-7-one was obtained on the general methods 1 from compound 1a in terms of beige crystalline solid, yield $70 \%$, mp $256{ }^{\circ} \mathrm{C}$, ${ }^{1} \mathrm{H}$ NMR (DMSO-d $\mathrm{d}_{6}, 400 \mathrm{MHz}$ ): 9.84 (1H, уш.c., $\mathrm{NH}), 3.05\left(3 \mathrm{H}, \mathrm{c}, \mathrm{CH}_{3}\right) ;{ }^{13} \mathrm{C}$ NMR (DMSO-d $\left.\mathrm{d}_{6}, 100 \mathrm{MHz}\right): 169.09\left(\mathrm{C}_{2}\right)$, $157.32\left(\mathrm{C}_{3 \mathrm{a}}\right), 144.05\left(\mathrm{C}_{7}\right), 143.57\left(\mathrm{C}_{6}\right)$, $25.58\left(\mathrm{CH}_{3} \mathrm{SO}\right)$; IR $\left(v / \mathrm{sm}^{-1}\right): 1750(\mathrm{C}=\mathrm{O})$; 1036 (-SO-); 1553,1340 ( $\left.\mathrm{NO}_{2}\right)$; elemental analysis, calculated for $\mathrm{C}_{5} \mathrm{H}_{4} \mathrm{~N}_{6} \mathrm{O}_{4} \mathrm{~S} \%$ : C $24.59, \mathrm{H} 1.65, \mathrm{~N}$ 34.42. Found, \%: C 24.62, H 1.43, N 34.28.

2-Ethylsulfinyl-6-nitro-1,2, 4 triazolo[5,1-c]1,2,4-triazine-7-one was obtained on the general methods 1 from compound $1 \mathrm{~b}$ in terms of beige crystalline solid. Yield $74 \%$, mp $227{ }^{\circ} \mathrm{C},{ }^{1} \mathrm{H}$ NMR (DMSO-d, $\left.6_{6} 400 \mathrm{MHz}\right): 11.16(1 \mathrm{H}$, br.s., $\mathrm{NH}), 3.36-3.16\left(2 \mathrm{H}, \mathrm{m}, \mathrm{CH}_{2}\right), 1.24$ $\left(3 \mathrm{H}, \mathrm{t}, J=7.4, \mathrm{CH}_{3}\right) ;{ }^{13} \mathrm{C}$ NMR (DMSO$\left.\mathrm{d}_{6}, 100 \mathrm{MHz}\right): 168.34\left(\mathrm{C}_{2}\right), 157.58\left(\mathrm{C}_{3 \mathrm{a}}\right)$, $144.36\left(\mathrm{C}_{7}\right), 143.87\left(\mathrm{C}_{6}\right), 46.64\left(\mathrm{CH}_{2} \mathrm{SO}\right)$, $6.15\left(\mathrm{CH}_{3}\right)$; IR $\left(v / \mathrm{sm}^{-1}\right): 1748(\mathrm{C}=\mathrm{O})$; 1022 (-SO-); 1552, $1336\left(\mathrm{NO}_{2}\right)$; elemen- tal analysis, calculated for $\mathrm{C}_{6} \mathrm{H}_{6} \mathrm{~N}_{6} \mathrm{O}_{4} \mathrm{~S}$, \%: C 27.91, H 2.34, N 32.55. Found, \%: C 27.87, H 2.27, N 32.31 .

2-Propylsulfinyl-6-nitro-1,2,4triazolo[5,1-c]1,2,4-triazine-7-one was obtained on the general methods 1 from compound $1 \mathrm{c}$ in terms of beige crystalline solid. Yield $72 \%$ mp $222{ }^{\circ} \mathrm{C}$, ${ }^{1} \mathrm{H}$ NMR (DMSO- $\left.\mathrm{d}_{6}, 400 \mathrm{MHz}\right): 8.41(1 \mathrm{H}$, br.s., $\mathrm{NH}), 3.29-3.18\left(2 \mathrm{H}, \mathrm{m}, \mathrm{SOCH}_{2}\right)$, $1.78-1.55\left(2 \mathrm{H}, \mathrm{m}, \mathrm{CH}_{2}\right), 1.01(3 \mathrm{H}, \mathrm{t}, J=7.4$, $\mathrm{CH}_{3}$ ); ${ }^{13} \mathrm{C}$ NMR (DMSO-d $\mathrm{d}_{6}, 100 \mathrm{MHz}$ ): $168.19\left(\mathrm{C}_{2}\right), 157.87\left(\mathrm{C}_{3 \mathrm{a}}\right), 144.19\left(\mathrm{C}_{7}\right)$, $143.46\left(\mathrm{C}_{6}\right), 54.15\left(\mathrm{CH}_{2} \mathrm{SO}\right), 15.21\left(\mathrm{CH}_{2}\right)$, $12.94\left(\mathrm{CH}_{3}\right) ; \mathrm{IR}\left(\mathrm{v} / \mathrm{sm}^{-1}\right): 1748(\mathrm{C}=\mathrm{O})$; 1013 (-SO-); 1556, $1336\left(\mathrm{NO}_{2}\right)$; elemental analysis, calculated for $\mathrm{C}_{7} \mathrm{H}_{8} \mathrm{~N}_{6} \mathrm{O}_{4} \mathrm{~S}, \%$ : $\mathrm{C} 30.88, \mathrm{H}$ 2.96, N 30.87. Found, \%: C 30.82, H 3.12, N 30.77 .

2-iso-Propylsulfinyl-6-nitro-1,2,4triazolo[5,1-c]1,2,4-triazine-7-one was obtained on the general methods 1 from compound $1 \mathrm{~d}$ in terms of beige crystalline solid. Yield $77 \%$ mp $239^{\circ} \mathrm{C}$, ${ }^{1} \mathrm{H}$ NMR (DMSO- $\left.\mathrm{d}_{6}, 400 \mathrm{MHz}\right): 7.60(1 \mathrm{H}$, br.s., $\mathrm{NH}), 3.49-3.38(1 \mathrm{H}, \mathrm{m}, \mathrm{CH}), 1.27$ $\left(6 \mathrm{H}, \mathrm{dd}, J=6.8,2 \mathrm{CH}_{3}\right) ;{ }^{13} \mathrm{C}$ NMR (DMSO-d, $100 \mathrm{MHz}): 167.79\left(\mathrm{C}_{2}\right), 157.42$ $\left(\mathrm{C}_{3 \mathrm{a}}\right), 144.33\left(\mathrm{C}_{7}\right), 143.86\left(\mathrm{C}_{6}\right), 53.07$ (CHSO), $15.92\left(\mathrm{CH}_{3}\right), 14.59\left(\mathrm{CH}_{3}\right) ; \mathrm{IR}(\mathrm{v} /$ $\left.\mathrm{sm}^{-1}\right)$ : $1752(\mathrm{C}=\mathrm{O})$; $992\left(-\mathrm{SO}^{-}\right) ; 1555,1342$ $\left(\mathrm{NO}_{2}\right)$; elemental analysis, calculated for $\mathrm{C}_{7} \mathrm{H}_{8} \mathrm{~N}_{6} \mathrm{O}_{4} \mathrm{~S}, \%$ : C 30.88, H 2.96, N 30.87. Found, \%: C 31.07, H 2.95, N 30.89.

The general methods 2 for preparation of 2-alkylsulfonyl-6-nitro-1,2,4triazolo[5,1-c]1,2,4-triazin-7-ones (4a-d).

To a suspension $0.01 \mathrm{~mol}$ of sodium salt 2-alkylthio-6-nitro-1,2,4-triazolo[5,1c] 1,2,4-triazin-7-one (1) in $14 \mathrm{~mL}$ of trifluoroacetic acid by mixing dropwise was added $4 \mathrm{ml}$ (2 eq.) of $30 \%$ hydrogen peroxide in order that a temperature wasn't 
above $80{ }^{\circ} \mathrm{C}$. Then the reaction mass was mixed at a room temperature during 3 hours, the precipitate was filtered and crystallized from iso-propanol.

2-Methylculfonyl-6-nit ro 1, 2,4triazolo[5,1-c]1,2,4-triazine-7-one was obtained on the general methods 2 from compound 1a in terms of beige crystalline solid. Yield $66 \%$ mp $275^{\circ} \mathrm{C}$, ${ }^{1} \mathrm{H}$ NMR (DMSO-d $\left.{ }_{6}, 400 \mathrm{MHz}\right): 7.57(1 \mathrm{H}$, br.s., $\mathrm{NH}), 3.41\left(3 \mathrm{H}, \mathrm{s}, \mathrm{CH}_{3}\right) ;{ }^{13} \mathrm{C} \mathrm{NMR}$ (DMSO-d ${ }_{6}, 100 \mathrm{MHz}$ ): $163.86\left(\mathrm{C}_{2}\right), 158.41$ $\left(\mathrm{C}_{3 \mathrm{a}}\right), 144.69\left(\mathrm{C}_{7}\right), 143.64\left(\mathrm{C}_{6}\right), 41.84$ $\left(\mathrm{CH}_{3} \mathrm{SO}_{2}\right) ; \mathrm{IR}\left(\mathrm{v} / \mathrm{sm}^{-1}\right): 1759(\mathrm{C}=\mathrm{O}) ; 1347$, $1138\left(-\mathrm{SO}_{2}-\right) ; 1570,1323\left(\mathrm{NO}_{2}\right)$ ); ; elemental analysis, calculated for $\mathrm{C}_{5} \mathrm{H}_{4} \mathrm{~N}_{6} \mathrm{O}_{5} \mathrm{~S}, \%$ : C 23.08, H 1.55, N 32.30. Found, \%: C 23.21, H 1.31, N 32.33 .

2-Ethylsulfonyl-6-nit ro-1, 2,4 triazolo[5,1-c]1,2,4-triazine-7-one was obtained on the general methods 2 from compound $1 \mathrm{~b}$ in terms of beige crystalline solid. Yield $64 \%, \mathrm{mp} 259^{\circ} \mathrm{C}$, ${ }^{1} \mathrm{H}$ NMR (DMSO-d, $\left.600 \mathrm{MHz}\right): 9.71(1 \mathrm{H}$, br.s., $\mathrm{NH}$ ), 3.52 ( $2 \mathrm{H}$, qv., $\left.J=7.4, \mathrm{CH}_{2}\right), 1.33$ $\left(3 \mathrm{H}, \mathrm{t}, J=7.4, \mathrm{CH}_{3}\right) ;{ }^{13} \mathrm{C}$ NMR (DMSO$\left.\mathrm{d}_{6}, 100 \mathrm{MHz}\right): 162.82\left(\mathrm{C}_{2}\right), 158.93\left(\mathrm{C}_{3 \mathrm{a}}\right)$, $145.06\left(\mathrm{C}_{7}\right), 144.02\left(\mathrm{C}_{6}\right), 48.54\left(\mathrm{CH}_{2} \mathrm{SO}_{2}\right)$, $7.25\left(\mathrm{CH}_{3}\right) ; \operatorname{IR}\left(v / \mathrm{sm}^{-1}\right): 1759(\mathrm{C}=\mathrm{O}) ; 1311$, $1140\left(-\mathrm{SO}_{2}{ }^{-}\right) ; 1557,1332\left(\mathrm{NO}_{2}\right)$; elemental analysis, calculated for $\mathrm{C}_{6} \mathrm{H}_{6} \mathrm{~N}_{6} \mathrm{O}_{5} \mathrm{~S}, \%$ : C 26.28, H 2.21, N 30.65. Found, \%: C 26.44, H 2.20, N 30.43 .

2-Propylsulfonyl-6-nitro-1,2,4triazolo[5,1-c]1,2,4-triazine-7-one was obtained on the general methods 2 from compound $1 \mathrm{c}$ in terms of beige crystalline solid. Yield $71 \%$, mp $264{ }^{\circ} \mathrm{C},{ }^{1} \mathrm{H}$ NMR (DMSO-d $\left.\sigma_{6}, 400 \mathrm{MHz}\right): 11.98(1 \mathrm{H}$, br.s., $\mathrm{NH}), 3.53\left(2 \mathrm{H}, \mathrm{t}, J=7.6, \mathrm{SO}_{2} \mathrm{CH}_{2}\right)$, 1.76-1.67 (2H, m, $\left.\mathrm{CH}_{2}\right), 0.98(3 \mathrm{H}, \mathrm{t}$, $\left.J=7.4, \mathrm{CH}_{3}\right) ;{ }^{13} \mathrm{C}$ NMR (DMSO-d ${ }_{6}$, $100 \mathrm{MHz}): 163.22\left(\mathrm{C}_{2}\right), 158.44\left(\mathrm{C}_{3 \mathrm{a}}\right)$,
$144.91\left(\mathrm{C}_{7}\right), 143.99\left(\mathrm{C}_{6}\right), 55.35\left(\mathrm{CH}_{2} \mathrm{SO}_{2}\right)$, $16.20\left(\mathrm{CH}_{2}\right), 12.94\left(\mathrm{CH}_{3}\right)$; IR $\left(\mathrm{v} / \mathrm{sm}^{-1}\right)$ : $1748(\mathrm{C}=\mathrm{O}) ; 1293,1139\left(-\mathrm{SO}_{2}^{-}\right) ; 1556$, $1326\left(\mathrm{NO}_{2}\right)$; elemental analysis, calculated for $\mathrm{C}_{7} \mathrm{H}_{8} \mathrm{~N}_{6} \mathrm{O}_{5} \mathrm{~S}, \%$ : C 29.17, H 2.80, N 29.16. Found, \%: C 29.01, H 2.88, N 29.24.

2-iso-Propylsulfinyl-6-nitro-1,2,4triazolo[5,1-c]1,2,4-triazine-7-one

(4d) was obtained on the general methods 2 from compound $1 \mathrm{~d}$ in terms of beige crystalline solid. Yield $62 \%$, mp $282{ }^{\circ} \mathrm{C}$, ${ }^{1} \mathrm{H}$ NMR (DMSO-d, $\left.400 \mathrm{MHz}\right): 8.27(1 \mathrm{H}$, br.s., $\mathrm{NH}), 3.73-3.63(1 \mathrm{H}, \mathrm{m}, \mathrm{CH}), 1.35$ $\left(6 \mathrm{H}, \mathrm{d}, J=6.8,2 \mathrm{CH}_{3}\right) ;{ }^{13} \mathrm{C}$ NMR (DMSO- $\left.\mathrm{d}_{6}, 100 \mathrm{MHz}\right): 161.91\left(\mathrm{C}_{2}\right), 158.80$ $\left(\mathrm{C}_{3 \mathrm{a}}\right), 144.98\left(\mathrm{C}_{7}\right), 144.02\left(\mathrm{C}_{6}\right), 54.30$ $\left(\mathrm{CHSO}_{2}\right), 15.02\left(2 \mathrm{CH}_{3}\right) ; \mathrm{IR}\left(\mathrm{v} / \mathrm{sm}^{-1}\right): 1749$ $(\mathrm{C}=\mathrm{O}) ; 1311,1135\left(-\mathrm{SO}_{2}^{-}\right) ; 1556,1326$ $\left(\mathrm{NO}_{2}\right)$; elemental analysis, calculated for $\mathrm{C}_{7} \mathrm{H}_{8} \mathrm{~N}_{6} \mathrm{O}_{5} \mathrm{~S}, \%$ : C 29.17, H 2.80, N 29.16. Found, \%: C 29.11, H 2.69, N 29.00.

The general methods 3 of nucleophilic replacement in 2-methylsulfonyl-6-nitro-1,2,4-triazolo[5,1-c]1,2,4-triazin-7-one.

To a suspension $0.001 \mathrm{~mol}$ of cysteine (or cysteamine) in $20 \mathrm{ml}$ of methanol were added triethylamine and were mixed at the argon atmosphere during $5 \mathrm{~min}$, then to a reaction mass were added equivalent of 2-alkylsulfonyl-triazolotriazin and were refluxed. The end of reaction is determined by TLC in system: butanolacetic acid-water 4:1:1. The resulting solid precipitate was washed with iso-propanol.

Triethylammonium salt of (2'-amino-2'-carboethoxyethylthio)6-nitro-1,2,4-triazolo[5,1-c] triazine hydrochloride (5) was obtained with use of the general methods 3 and 3 equivalent of triethylamine. The product was crystallized from the water ethanol in terms of canary crystalline solid. Yield $46 \%$, 
mp $158{ }^{\circ} \mathrm{C},{ }^{1} \mathrm{H}$ NMR $\left(\mathrm{D}_{2} \mathrm{O}, 400 \mathrm{MHz}\right)$ : $4.27(1 \mathrm{H}, \mathrm{dd}, J=7.5,3.8, \mathrm{CHN}), 3.98$ $\left(1 \mathrm{H}, \mathrm{dd}, J=15.2,3.8, \mathrm{H}_{\mathrm{a}}\right.$ in $\left.\mathrm{SCH}_{2}\right), 3.66$ $\left(1 \mathrm{H}, \mathrm{dd}, J=15.2,7.5, \mathrm{H}_{\mathrm{b}}\right.$ in $\left.\mathrm{SCH}_{2}\right), 3.21$ $\left(6 \mathrm{H}\right.$, qv., $\left.J=7.28,3 \mathrm{CH}_{2}\right), 1.29(9 \mathrm{H}, \mathrm{t}, J=$ $\left.7.28,3 \mathrm{CH}_{3}\right) ;{ }^{13} \mathrm{C}$ NMR $\left(\mathrm{D}_{2} \mathrm{O}, 100 \mathrm{MHz}\right)$ : $172.02\left(\mathrm{COO}^{-}\right), 166.09\left(\mathrm{C}_{2}\right), 159.16\left(\mathrm{C}_{3 \mathrm{a}}\right)$, $144.55\left(\mathrm{C}_{7}\right), 143.06\left(\mathrm{C}_{6}\right), 54.47(\mathrm{CHN})$, $46.71\left(3 \mathrm{CH}_{2}\right), 31.67\left(\mathrm{SCH}_{2}\right), 8.27\left(3 \mathrm{CH}_{3}\right)$; IR $\left(v / \mathrm{sm}^{-1}\right): 1682,1615(\mathrm{C}=\mathrm{O}) ; 1504,1361$ $\left(\mathrm{NO}_{2}\right)$; elemental analysis, calculated for $\mathrm{C}_{13} \mathrm{H}_{22} \mathrm{~N}_{8} \mathrm{O}_{5} \mathrm{~S}^{*} \mathrm{H}_{2} \mathrm{O}, \%$ : C 37.10, H 5.71, N 26.63. Found, \%: C 36.96, H 5.56, N 26.72.

2-(2'-Amino-ethylthio)-6-nitro-1,2,4triazolo[5,1-c] $1,2,4$-triazine hydrate (6) was obtained with use of the general methods 3 and 2 equivalent of triethylamine. The product was crystallized from the water ethanol in terms of canary crystalline solid. Yield $41 \%$, mp $285^{\circ} \mathrm{C}$, ${ }^{1} \mathrm{H}$ NMR ( $\left.\mathrm{D}_{2} \mathrm{O}, 400 \mathrm{MHz}\right): 3.58(2 \mathrm{H}, \mathrm{t}$, $\left.J=7.03, \mathrm{NCH}_{2}\right), 3.49\left(2 \mathrm{H}, \mathrm{t}, J=7.03, \mathrm{SCH}_{2}\right)$; ${ }^{13} \mathrm{C}$ NMR (DMSO- $\mathrm{d}_{6}, 100 \mathrm{MHz}$ ): 163.95 $\left(\mathrm{C}_{2}\right), 160.12\left(\mathrm{C}_{3 \mathrm{a}}\right), 144.77\left(\mathrm{C}_{7}\right), 142.94\left(\mathrm{C}_{6}\right)$, $38.69\left(\mathrm{CH}_{2} \mathrm{~N}\right), 27.87\left(\mathrm{SCH}_{2}\right) ; \mathrm{IR}\left(\mathrm{v} / \mathrm{sm}^{-1}\right)$ : $1690(\mathrm{C}=\mathrm{O}) ; 1514,1371\left(\mathrm{NO}_{2}\right) ; 3521$ (broadened) $\left(-\mathrm{NH}_{3}^{+}\right)$; elemental analysis, calculated for $\mathrm{C}_{6} \mathrm{H}_{7} \mathrm{~N}_{7} \mathrm{O}_{3} \mathrm{~S}^{*} \mathrm{H}_{2} \mathrm{O}, \%$ : $\mathrm{C}$ 26.18, H 3.27, N 35.64. Found, \%: C 25.91, H 2.98, N 35

1. Rusinov V. L., Ulomskii E. N., Chupakhin O. N., Charushin V.N. Azolo[5,1-c]-1,2,4-triazines as a new class of antiviral compounds. Russ. Chem. Bull., Int. Ed. 2008; 57(5): 985-1014. DOI: $10.1007 / \mathrm{s} 11172-008-0130-8$.

2. Karpenko I., Deev S., Kiselev O., Charushin V., Rusinov V., Ulomsky E., Deeva E., Yanvarev D., Ivanov A., Smirnova O., Kochetkov S., Chupakhin O., Kukhanova M. Antiviral properties, metabolism, and pharmacokinetics of a novel azolo-1,2,4-triazine-derived inhibitor of influenza A and B virus replication. Antimicrobial Agents and Chemotherapy. 2010; 54(5): 2017-2022. DOI: 10.1128/AAC.01186-09.

3. Denicola A., Radi R. Peroxynitrite and drug-dependent toxicity. Toxicology. 2005; 208(2): 273-288. DOI: 10.1016/j.tox.2004.11.023.

4. Liu J., Dang Q., Wei Z., Zhang H., Bai X. Parallel solution-phase synthesis of a 2,6,8,9-tetrasubstituted purine library via a sulfur intermediate. J. of Combinatorial Chemistry. 2005; 7(4): 627-636. DOI: 10.1021/cc049819p.

5. Rusinov V. L., Ulomskii E. N., Chupakhin O.N., Petrov A. Yu., Sharonov E. A. Nitroazines. 9. Characteristic features of nucleophilic substitution of the nitro group in dihydroazolo[5,1-c] [1,2,4]triazines. Chemistry of Heterocyclic Compounds. 1989; 25(2): 209-213. DOI: 10.1007/BF00479921. 


\author{
Н. Р. Медведева ${ }^{1}$, И. С. Сапожникова ${ }^{1}$, \\ В. Л. Русинов ${ }^{1,2}$, Е. Н. Уломский ${ }^{1}$ \\ ${ }^{1}$ Уральский федеральный университет \\ 620002 Екатеринбург, ул. Мира, 19. \\ E-mail: nataly-medvedeva@yandex.ru \\ ${ }^{2}$ Институт органического синтеза УрО РАН \\ 620990 Екатеринбург ул. С. Ковалевской, 22 / \\ Академическая, 20. \\ E-mail:v.l.rusinov@urfu.ru
}

\title{
Редокс превращения и нуклеофильные замещения как возможные метаболические реакции препарата «Триазавирина». Химическое моделирование процессов метаболизма
}

В качестве модельных метаболических превращений противовирусного препарата «Триазавирин» и его аналогов - 2-алкилтио-6-нитро-1,2,4-триазоло-[5,1-c][1,2,4]триазин-7-онов 1a-d рассмотрены реакции окисления алкитиогруппы с образованием соответствующих сульфоксидов 2a-d и сульфонов 3a-d, а также процесс нуклеофильного замещения сульфонильной группы цистеином и цистеамином с образованием соединений 5 и 6.

Ключевые слова: биологически активные соединения, гетероциклы, триази, триазаверин, антивирусные препараты.

() Медведева Н. Р., Сапожникова И. С., Русинов В. Л., Уломский Е. Н., 2015

\section{Введение}

Актуальность создания новых противовирусных средств обусловлена совместным действием таких постоянно действующих факторов, как распространение социально значимых, особо опасных инфекций, а также появление патогенных вирусных штаммов, устойчивых к действию существующих лекарственных средств.

Противовирусный препарат «Триазавирин» и его аналоги обладают высокой эффективностью в опытах in

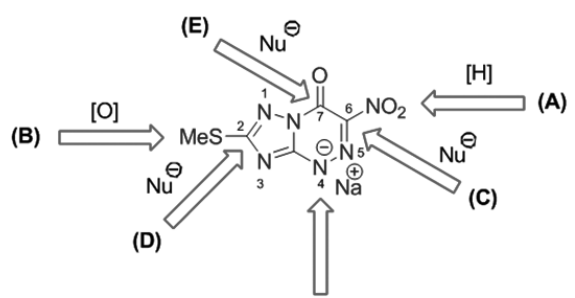

(F)

vivo [1] и пониженной активностью в экспериментах на клеточных культурах, что позволяет предположить противовирусное действие не самого 
«Триазавирина», а продуктов его превращений в организме. Одним из способов выявления таких превращений является прогнозирование возможных продуктов модификаций соединений и химический синтез моделей.

Исходя из молекулярной структуры соединения 1a и изучения химических свойств нитроазоло[5,1-c][1,2,4] триазинов, можно предположить различные варианты метаболизма [1]. Редокс-превращения в организме: (направления $A, B$ ) как восстановление нитрогруппы под действием редуктаз (направление $A$ ); окисление алкилтиофрагмента под действием оксидаз (направление B) и его дальнейшие превращения. Участие трансфераз при действии N- и S-нуклеофилов, таких, например, как лизин, аргинин, цистеин способно приводить к замещению алкилтио- или нитрогрупп (направления $C, D$ ).

Гидролитические ферменты также вполне способны обусловливать превращения «Триазавирина"», сопровождающиеся деструкцией триазинового цикла с разрывом связи C-N (направление E). Возможно и алкилирование по атому азота (направление $F$ ).

Что касается редокс-превращений «Триазавирина», то ранее нами было установлено, что при внутрижелудочном ведении лабораторным животным субстанции препарата происходит восстановление нитрогруппы с образованием - 2-метилтио-6-амино-1,2,4триазоло [5,1-c][1,2,4] триазин-7-она [2], не проявляющего противовирусного действия в экспериментах in vitro и, скорее всего, не являющегося действующим метаболитом.

\section{Результаты и обсуждение}

Рассматривая возможность окисления алкилтиогрупп при использовании «Триазавирина » и его аналогов, нужно отметить, что при большинстве вирусных инфекций уже на ранней стадии процесса происходит усиление генерирования активных форм кислорода [3]. Исходя из изложенного естественно предположить, что в этих условиях могут происходить реакции окисления S-метильной группы «Триазавирина», свойственные, например, для $\mathrm{SH}$-содержащей аминокислоты метионина при окислительном стрессе.

В настоящем сообщении приводятся данные о постановке модель- ных экспериментов с препаратом «Триазавирин» - изучение окисления $\mathrm{S}$-метильной группы и поведения продуктов окисления под действием $\mathrm{S}$-нуклеофилов. Такой подход позволяет, с одной стороны, предсказать течение химических реакций, сопутствующих поведению препарата в организме, а с другой стороны, путем синтеза соответствующих соединений смоделировать процесс образования ковалентных связей азоло[5,1-с]-1,2,4триазинов с S-фрагментами ключевых белков как клетки, так и вируса.

\section{Редокс-превращения «Триазавирина» и его производных}

Первым аспектом работы стал синтез модельных соединений - окисление сульфгидрильной группы в молекулах натриевых солей 2-алкилтио-6-ни- 
тро-1,2,4-триазоло[5,1-с]-1,2,4-триазин-7-онов 1a-d или сопряженных с ними N-H кислот 2a-d, включающее образование гетероциклических сульфоксидов 3a-d и сульфонов 4a-d. При обработке соединений 1a-d или 2a-d эквимолярным количеством 18 \%-го пероксида водорода в трифторуксусной кислоте получены сульфоксидоы 3a-d (схема 1).
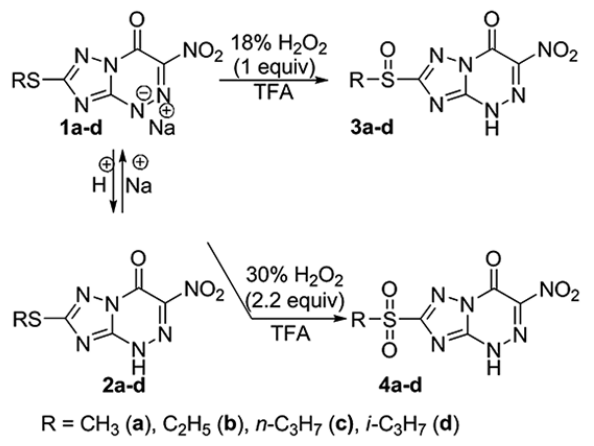

Схема 1

Дальнейшее окисление до соответствующих сульфонов 4a-d с выходами 62-71\% осуществлено постепенным прибавлением избытка 2,2 эквивалента $30 \% \mathrm{H}_{2} \mathrm{O}_{2}$ к суспензии 2-алкилтио- 1,2,4-триазоло [5,1-c]триазинов 1a-d в трифторуксусной кислоте при комнатной температуре.

Представленные реакции моделируют возможные метаболические превращения «Триазавирина» и его производных под действием активных форм кислорода, включая $\mathrm{H}_{2} \mathrm{O}_{2}$.

Нуклеофильное замещение метилсульфонильной группы. Известно, что алкилсульфонильные фрагменты восприимчивы к реакциям замещения при взаимодействии с нуклеофилами [4]. Что касается поведения нитрогруппы в «Триазавирине», то хорошо известна склонность $\mathrm{NO}_{2}$-группы, связанной с ароматическим (гетероароматическим) циклом к замещению под действием нуклеофилов [5].

Таким образом, в структуре 2-алкилсульфонил - 1,2,4 - триазоло - [5, 1 c]-1,2,4-триазин-7-онов 4a-d присутствуют две группировки, склонные к нуклеофильному вытеснению. Эта ситуация представляет интерес для исследования сравнительной реакционной способности легкоуходящих групп - $\mathrm{CH}_{3} \mathrm{SO}_{2}$ - в 1,2,4-триазольном и $\mathrm{NO}_{2}^{-}$в 1,2,4-триазиновом циклах в соединениях 4a-d, а также как модель поведения «Триазавирина» в организме. В качестве нуклеофильных реагентов в настоящей работе использовались S-нуклеофилы (цистеин и цистеамин), которые можно рассматривать как модели фрагментов белков, содержащих цистеиновый фрагмент.

Замещение сульфонильного фрагмента в 2-метилсульфонил-1,2,4триазоло[5,1-с]-1,2,4-триазине 4 а под действием как цистеина, так и цистеамина происходит при кипячении в сухом метаноле в присутствии триэтиламина в течение нескольких дней с образованием соединений 5 и 6 продуктов замещения метилсульфонильной группы с выходами 41-46 \% (схема 2).

Данные ЯМР, ИК-спектроскопии и элементного анализа для соединений

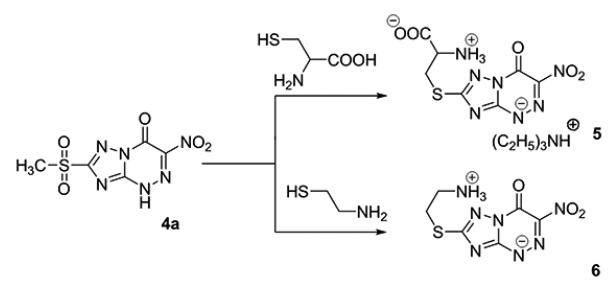

Схема 2 
5, 6 соответствуют приписываемым структурам.

Описанное замещение алкилсульфонильной группы в соединении $\mathbf{4 a}$

\section{Экспериментальная часть}

Спектры ЯМР ${ }^{1} \mathrm{H}$ и ${ }^{13} \mathrm{C}$ получены на приборе Bruker DRX-400 (400 МГц для ${ }^{1} \mathrm{H}$ и 100 МГц для ${ }^{13} \mathrm{C}$ ) в ДМСО$\mathrm{d}_{6}$, и $\mathrm{D}_{2} \mathrm{O}$, химические сдвиги приведены в $\delta$-шкале относительно внутреннего стандарта для спектров $1 \mathrm{H}$ ЯМР - ТМС. Элементные анализы (C, $\mathrm{H}, \mathrm{N})$ были проведены на анализатоpe «Perkin Elmer 2400-II CHNS/O». ИК

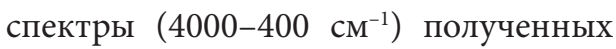
соединений записаны на спектрометре «Perkin Elmer Spectrum One B FTIR» в тонком слое образца (DRA). Контроль за ходом реакций и чистотой синтезированных соединений осуществляли с помощью ТСХ на пластинках Sorbfil (фирма 3AO «Сорбполимер») в системах этилацетат и бутанол-уксусная кислота-вода 4:1:1.

Общая методика 1 получения 2 -алкил сульфинил-6-нитро- $1,2,4$ триазоло[5,1-c]-1,2,4-триазин-7-онов (3a-d). К суспензии 0,01 моль натриевой соли 2-алкилтио-6-нитро-1,2,4триазоло[5,1-c] 1,2,4-триазин-7-она (1) в 10 мл трифторуксусной кислоты при перемешивании добавляют 1,78 мл (1 экв) 18 \%-й перекиси водорода. Реакционную массу перемешивают при комнатной температуре три часа, осадок отфильтровывают и кристаллизуют из изо-пропанола.

2-Метилсульфинил-6-нитро- 1,2,4триазоло[5,1-c]1,2,4-триазин-7-он (3a) был получен из соединения 1a c использованием общей методики 1 в виде бежевых кристаллов, выход 70 \%, mp является аргументами в пользу предположения о возможном участии этого фрагмента в метаболических превращениях «Триазавирина».

$256{ }^{\circ} \mathrm{C},{ }^{1} \mathrm{H}$ NMR (DMSO-d, $400 \mathrm{MHz}$ ): 9,84 (1H, уш.с., NH), 3,05 (3H, c, CH3); ${ }^{13} \mathrm{C}$ NMR (DMSO-d, $100 \mathrm{MHz}$ ): 169,09 $\left(\mathrm{C}_{2}\right), 157,32\left(\mathrm{C}_{3 \mathrm{a}}\right), 144,05\left(\mathrm{C}_{7}\right), 143,57\left(\mathrm{C}_{6}\right)$, 25,58 ( $\left.\mathrm{CH}_{3} \mathrm{SO}\right) ; \mathrm{IR}\left(v / \mathrm{sm}^{-1}\right): 1750(\mathrm{C}=\mathrm{O})$; 1036 (-SO-); 1553,1340 ( $\left.\mathrm{NO}_{2}\right)$; Элемент. анализ $\mathrm{C}_{5} \mathrm{H}_{4} \mathrm{~N}_{6} \mathrm{O}_{4} \mathrm{~S}$, вычислено \%: C 24,59, Н - 1,65, N - 34,42, найдено, \%: C - 24,62, H - 1,43, N - 34,28.

2-Этил сульфинил- 6 - нитр о- 1, 2, 4 триазоло[5,1-c] 1,2,4-триазин-7-он (3b) был получен из соединения $\mathbf{1 b}$ с использованием общей методики 1 в виде бежевых кристаллов, выход $74 \%$, mp $227{ }^{\circ} \mathrm{C},{ }^{1} \mathrm{H}$ NMR (DMSO-d, $400 \mathrm{MHz}$ ): 11,16 (1H, уш.с., NH), 3,36-3,16 (2H, м, $\left.\mathrm{CH}_{2}\right), 1,24\left(3 \mathrm{H}, \mathrm{T}, J=7,4, \mathrm{CH}_{3}\right) ; 13 \mathrm{C} \mathrm{NMR}$ (DMSO-d, $100 \mathrm{MHz}$ ): 168,34 ( $\left.\mathrm{C}_{2}\right), 157,58$ $\left(\mathrm{C}_{3 \mathrm{a}}\right), 144,36\left(\mathrm{C}_{7}\right), 143,87 \quad\left(\mathrm{C}_{6}\right), 46,64$ $\left(\mathrm{CH}_{2} \mathrm{SO}\right), 6,15\left(\mathrm{CH}_{3}\right) ; \mathrm{IR}\left(\mathrm{v} / \mathrm{sm}^{-1}\right): 1748$ $(\mathrm{C}=\mathrm{O}) ; 1022$ (-SO-); 1552, $1336\left(\mathrm{NO}_{2}\right)$; Элемент анализ $\mathrm{C}_{6} \mathrm{H}_{6} \mathrm{~N}_{6} \mathrm{O}_{4} \mathrm{~S}$, вычислено \%: C - 27,91, H - 2,34, N - 32,55, найдено, \%: C - 27,87, H - 2,27, N - 32,31.

2-Пропилсульфинил-6-нитро-1,2,4триазоло[5,1-с]1,2,4-триазин-7-он (3c) был получен из соединения 1с с использованием общей методики 1 в виде бежевых кристаллов, выход $72 \%$, mp $222{ }^{\circ} \mathrm{C},{ }^{1} \mathrm{H}$ NMR (DMSO-d, $400 \mathrm{MHz}$ ): 8,41 (1H, уш.с., NH), 3,29-3,18 (2H, м, $\left.\mathrm{SOCH}_{2}\right), 1,78-1,55\left(2 \mathrm{H}, \mathrm{m}, \mathrm{CH}_{2}\right), 1,01$ $\left(3 \mathrm{H}, \mathrm{T}, J=7,4, \mathrm{CH}_{3}\right) ;{ }^{13} \mathrm{C}$ NMR (DMSO$\left.\mathrm{d}_{6}, 100 \mathrm{MHz}\right): 168,19\left(\mathrm{C}_{2}\right), 157,87\left(\mathrm{C}_{3 \mathrm{a}}\right)$, $144,19\left(\mathrm{C}_{7}\right), 143,46\left(\mathrm{C}_{6}\right), 54,15\left(\mathrm{CH}_{2} \mathrm{SO}\right)$, $15,21\left(\mathrm{CH}_{2}\right), 12,94\left(\mathrm{CH}_{3}\right) ; \mathrm{IR}\left(\mathrm{v} / \mathrm{sm}^{-1}\right)$ : $1748(\mathrm{C}=\mathrm{O}) ; 1013\left(-\mathrm{SO}^{-}\right) ; 1556,1336$ 
$\left(\mathrm{NO}_{2}\right)$; Элементный анализ $\mathrm{C}_{7} \mathrm{H}_{8} \mathrm{~N}_{6} \mathrm{O}_{4} \mathrm{~S}$, вычислено \%: С - 30,88, Н - 2,96, $\mathrm{N}$ 30,87 , найдено, \%: C - 30,82, Н - 3,12, $\mathrm{N}-30,77$.

2 - изо - Пропилсульфинил - 6 - ни тро-1,2,4-триаз оло $[5,1$-с]-1,2,4-триазин-7-он (3d) был получен из соединения 1d с использованием общей методики 1 в виде бежевых кристаллов, выход $77 \%$, mp $239{ }^{\circ} \mathrm{C},{ }^{1} \mathrm{H}$ NMR (DMSO-d, $400 \mathrm{MHz}$ ): 7,60 (1H, уш.c., $\mathrm{NH}), 3,49-3,38(1 \mathrm{H}, \mathrm{m}, \mathrm{CH}), 1,27$ (6H, дд, $\left.J=6,8,2 \mathrm{CH}_{3}\right) ;{ }^{13} \mathrm{C}$ NMR (DMSO-d, 100 $\mathrm{MHz}): 167,79\left(\mathrm{C}_{2}\right), 157,42\left(\mathrm{C}_{3 \mathrm{a}}\right), 144,33$ $\left(\mathrm{C}_{7}\right), 143,86\left(\mathrm{C}_{6}\right), 53,07$ (CHSO), 15,92 $\left(\mathrm{CH}_{3}\right), 14,59\left(\mathrm{CH}_{3}\right) ; \mathrm{IR}\left(\mathrm{v} / \mathrm{sm}^{-1}\right): 1752$ $(\mathrm{C}=\mathrm{O}) ; 992$ (-SO-); 1555, $1342\left(\mathrm{NO}_{2}\right)$; Элементный анализ $\mathrm{C}_{7} \mathrm{H}_{8} \mathrm{~N}_{6} \mathrm{O}_{4} \mathrm{~S}$, вычислено \%: C - 30,88, Н - 2,96, N - 30,87, найдено, \%: С - 31,07, Н - 2,95, N 30,89 .

Общая методика 2 получения 2 -алкилсульфонил - 6 - ни тро- 1, 2, 4триазоло[5,1-c]-1,2,4-триазин-7-онов (4a-d). К суспензии 0,01 моль натриевой соли 2-алкилтио-6-нитро-1,2,4триазоло [5,1-c]-1,2,4-триазин-7-она (1) в 14 мл трифторуксусной кислоты при перемешивании по каплям добавляют 4 мл (2 экв) $30 \%$ перекиси водорода так, чтобы температура не превышала $80{ }^{\circ} \mathrm{C}$. Далее реакционную массу перемешивают при комнатной температуре еще три часа, осадок отфильтровывают и кристаллизуют из изо-пропанола.

2-Метилсульфонил-6-нитро- $1,2,4$ триазоло [5,1-c]-1,2,4-триазин-7-он (4a) был получен из соединения 1a с использованием общей методики 2 в виде бежевых кристаллов, выход $66 \%, \mathrm{mp} 275{ }^{\circ} \mathrm{C},{ }^{1} \mathrm{H}$ NMR (DMSO-d, $400 \mathrm{MHz}$ ): 7,57 (1H, уш.с., NH), 3,41 (3H, c, $\left.\mathrm{CH}_{3}\right) ;{ }^{13} \mathrm{C}$ NMR (DMSO-d, $\left.100 \mathrm{MHz}\right)$ : $163,86\left(\mathrm{C}_{2}\right), 158.41\left(\mathrm{C}_{3 \mathrm{a}}\right), 144,69\left(\mathrm{C}_{7}\right)$, $143,64 \quad\left(\mathrm{C}_{6}\right), \quad 41,84 \quad$ (CH3SO2); IR $\left(v / \mathrm{sm}^{-1}\right): 1759 \quad(\mathrm{C}=\mathrm{O}) ; 1347,1138$ $\left(-\mathrm{SO}_{2}^{-}\right) ; 1570,1323\left(\mathrm{NO}_{2}\right)$; Элементный анализ $\mathrm{C}_{5} \mathrm{H}_{4} \mathrm{~N}_{6} \mathrm{O}_{5} \mathrm{~S}$, вычислено \%: C $23,08, \mathrm{H}-1,55, \mathrm{~N}$ - 32,30, найдено, \%: $\mathrm{C}-23,21, \mathrm{H}-1,31, \mathrm{~N}-32,33$.

2-Этилсульфонил-6-нитр - $1,2,4$ триазоло[5,1-c]-1,2,4-триазин-7-он (4b) был получен из соединения $\mathbf{1 b}$ с использованием общей методики 2 в виде бежевых кристаллов, выход $64 \%$, mp $259{ }^{\circ} \mathrm{C},{ }^{1} \mathrm{H}$ NMR (DMSO-d, $400 \mathrm{MHz}$ ): 9,71 (1H, уш.с., $\mathrm{NH}), 3,52$ (2H, кв., $J=$ $\left.7,4, \mathrm{CH}_{2}\right), 1,33\left(3 \mathrm{H}, \mathrm{T}, J=7,4, \mathrm{CH}_{3}\right) ; 13 \mathrm{C}$ NMR (DMSO-d, $100 \mathrm{MHz}$ ): 162,82 $\left(\mathrm{C}_{2}\right), 158,93\left(\mathrm{C}_{3 \mathrm{a}}\right), 145,06\left(\mathrm{C}_{7}\right), 144,02$ $\left(\mathrm{C}_{6}\right), 48,54\left(\mathrm{CH}_{2} \mathrm{SO}_{2}\right), 7,25\left(\mathrm{CH}_{3}\right)$; IR $(v /$ $\left.\mathrm{sm}^{-1}\right)$ : 1759 (C=O); 1311, 1140 (-SO2-); 1557, $1332\left(\mathrm{NO}_{2}\right)$; Элементный анализ $\mathrm{C}_{6} \mathrm{H}_{6} \mathrm{~N}_{6} \mathrm{O}_{5} \mathrm{~S}$, вычислено \%: C - 26,28, $\mathrm{H}$ $2,21, \mathrm{~N}-30,65$, найдено, \%: C - 26,44, $\mathrm{H}-2,20, \mathrm{~N}-30,43$.

2-Пропилсульфонил-6-нитро-1,2,4триазоло [5, 1-с]- 1, 2, 4-триазин-7-он (4c) был получен из соединения 1c c использованием общей методики 2 в виде бежевых кристаллов, выход $71 \%, \mathrm{mp} 264{ }^{\circ} \mathrm{C},{ }^{1} \mathrm{H}$ NMR (DMSO- $\mathrm{d}_{6}$, $400 \mathrm{MHz}$ ): 11,98 (1H, уш.c., NH), 3,53 $\left(2 \mathrm{H}, \mathrm{T}, J=7,6, \mathrm{SO}_{2} \mathrm{CH}_{2}\right), 1,76-1,67(2 \mathrm{H}, \mathrm{M}$, $\left.\mathrm{CH}_{2}\right), 0,98\left(3 \mathrm{H}, \mathrm{T}, \mathrm{J}=7,4, \mathrm{CH}_{3}\right) ;{ }^{13} \mathrm{C} \mathrm{NMR}$ (DMSO-d, $100 \mathrm{MHz}$ ): 163,22 (C $), 158,44$ $\left(\mathrm{C}_{3 \mathrm{a}}\right), 144,91 \quad\left(\mathrm{C}_{7}\right), 143,99 \quad\left(\mathrm{C}_{6}\right), 55,35$ $\left(\mathrm{CH}_{2} \mathrm{SO}_{2}\right), 16,20\left(\mathrm{CH}_{2}\right), 12,94\left(\mathrm{CH}_{3}\right)$; IR $\left(v / \mathrm{sm}^{-1}\right): 1748(\mathrm{C}=\mathrm{O}) ; 1293,1139\left(-\mathrm{SO}_{2}{ }^{-}\right)$; 1556, $1326\left(\mathrm{NO}_{2}\right)$; Элементный анализ $\mathrm{C}_{7} \mathrm{H}_{8} \mathrm{~N}_{6} \mathrm{O}_{5} \mathrm{~S}$, вычислено \%: C - 29,17, $\mathrm{H}$ 2,80, N - 29,16, найдено, \%: C - 29,01, $\mathrm{H}-2,88, \mathrm{~N}-29,24$.

2 - изо - Пропи и с ульфи нил - 6 - ни тро-1,2,4-триазоло $[5,1$-с]-1,2,4-триа- 
зин-7-он (4d) был получен из соединения $1 \mathrm{~d}$ с использованием общей методики 3 в виде бежевых кристаллов, выход $62 \%, \mathrm{mp} 282{ }^{\circ} \mathrm{C},{ }^{1} \mathrm{H}$ NMR (DMSO-d $\left.{ }_{6}, 400 \mathrm{MHz}\right): 8,27$ (1H, уш.c., $\mathrm{NH}), 3,73-3,63(1 \mathrm{H}$, м, $\mathrm{CH}), 1,35$ (6H, д, $\left.J=6,8,2 \mathrm{CH}_{3}\right) ;{ }^{13} \mathrm{C}$ NMR (DMSO- $\mathrm{d}_{6}, 100$ $\mathrm{MHz}): 161,91\left(\mathrm{C}_{2}\right), 158,80\left(\mathrm{C}_{3 \mathrm{a}}\right), 144,98$ $\left(\mathrm{C}_{7}\right), 144,02\left(\mathrm{C}_{6}\right), 54,30\left(\mathrm{CHSO}_{2}\right), 15,02$ $\left(2 \mathrm{CH}_{3}\right)$; IR $\left(v / \mathrm{sm}^{-1}\right): 1749(\mathrm{C}=\mathrm{O}) ; 1311$, $1135\left(-\mathrm{SO}_{2}-\right)$; 1556, $1326\left(\mathrm{NO}_{2}\right)$; Элементный анализ $\mathrm{C}_{7} \mathrm{H}_{8} \mathrm{~N}_{6} \mathrm{O}_{5} \mathrm{~S}$, вычислено \%: C - 29,17, H - 2,80, N - 29.16, найдено, \%: С - 29,11, Н - 2,69, N - 29,00.

Общая методика 3 нуклеофильного замещения в 2-метилсульфонил-6-нитро-1,2,4-триазоло [5,1-с]1,2,4-триазин-7-оне. К суспензии 0,001 моль цистеина (или цистеамина) в 20 мл метанола прибавляют триэтиламин и перемешивают в атмосфере аргона в течение пяти минут, затем в реакционную массу вносят эквивалент 2 -алкилсульфонил-триазолотриазина и кипятят. Окончание реакции определяют по ТСХ в системе бутанол-уксусная кислота-вода 4:1:1, после чего реакционную массу упаривают досуха. Полученный осадок промывают изопропиловым спиртом и очищают от примесей.

\section{Триаэтиламмониевую}

соль (2'-амино-2'-карбоксиэтилтио)-6-нитро-1,2,4-триазоло[5,1-c]-1,2,4-триазина гидрат (5) получают с использованием общей методики 3 и 3 экв. триаэтиламина. Продукт кристаллизуют из водного этанола в виде ярко-желтых кристаллов, выход $46 \%, \mathrm{mp} 158^{\circ} \mathrm{C},{ }^{1} \mathrm{H} \mathrm{NMR}$ $\left(\mathrm{D}_{2} \mathrm{O}, 400 \mathrm{MHz}\right): 4,27(1 \mathrm{H}$, дд, $J=7,5$, 3,8, CHN), 3,98 (1H, дд, $J=15,2,3,8, \mathrm{H}_{\mathrm{a}}$ в $\left.\mathrm{SCH}_{2}\right), 3,66\left(1 \mathrm{H}\right.$, дд, $J=15,2,7,5, \mathrm{H}_{\mathrm{b}}$ в $\left.\mathrm{SCH}_{2}\right), 3,21\left(6 \mathrm{H}\right.$, кв., $\left.J=7,28,3 \mathrm{CH}_{2}\right)$, $1,29\left(9 \mathrm{H}, \mathrm{T}, J=7,28,3 \mathrm{CH}_{3}\right) ; 13 \mathrm{C} \mathrm{NMR}$ $\left(\mathrm{D}_{2} \mathrm{O}, 100 \mathrm{MHz}\right): 172,02\left(\mathrm{COO}^{-}\right), 166,09$ $\left(\mathrm{C}_{2}\right), 159,16\left(\mathrm{C}_{3 \mathrm{a}}\right), 144,55\left(\mathrm{C}_{7}\right), 143,06\left(\mathrm{C}_{6}\right)$, $54,47(\mathrm{CHN}), 46,71\left(3 \mathrm{CH}_{2}\right), 31,67\left(\mathrm{SCH}_{2}\right)$, $8,27\left(3 \mathrm{CH}_{3}\right) ; \mathrm{IR}\left(\mathrm{v} / \mathrm{sm}^{-1}\right): 1682,1615$ $(\mathrm{C}=\mathrm{O}) ; 1504,1361\left(\mathrm{NO}_{2}\right)$; Элементный анализ $\mathrm{C}_{13} \mathrm{H}_{22} \mathrm{~N}_{8} \mathrm{O}_{5} \mathrm{~S} \times \mathrm{H}_{2} \mathrm{O}$, вычислено \%: C - 37,10, H - 5,71, N - 26,63, найдено, \%: С - 36,96, Н - 5,56, N - 26,72.

2-(2'-Амино-этилтио)-6-нитро-1,2,4триазоло[5,1-c]-1,2,4-триазин гидрат (6) получают с использованием общей методики 3 и 2 экв. триаэтиламина. Продукт кристаллизуют из водного метанола в виде ярко-желтых кристаллов, выход $41 \%, \mathrm{mp} 285{ }^{\circ} \mathrm{C}, 1 \mathrm{H}$ NMR $\left(\mathrm{D}_{2} \mathrm{O}, 400 \mathrm{MHz}\right): 3,58(2 \mathrm{H}, \mathrm{T}, J=$ $\left.7,03, \mathrm{NCH}_{2}\right), 3,49\left(2 \mathrm{H}, \mathrm{T}, J=7,03, \mathrm{SCH}_{2}\right)$; 13C NMR (DMSO-d $6100 \mathrm{MHz}$ ): 163,95 $\left(\mathrm{C}_{2}\right), 160,12\left(\mathrm{C}_{3 \mathrm{a}}\right), 144,77\left(\mathrm{C}_{7}\right), 142,94\left(\mathrm{C}_{6}\right)$, $38,69\left(\mathrm{CH}_{2} \mathrm{~N}\right), 27,87\left(\mathrm{SCH}_{2}\right)$; IR $\left(v / \mathrm{sm}^{-1}\right)$ : $1690(\mathrm{C}=\mathrm{O}) ; 1514,1371\left(\mathrm{NO}_{2}\right) ; 3521$ (broadened) $\left(-\mathrm{NH}_{3}^{+}\right)$; Элементный анализ $\mathrm{C}_{6} \mathrm{H}_{7} \mathrm{~N}_{7} \mathrm{O}_{3} \mathrm{~S} \times \mathrm{H}_{2} \mathrm{O}$, вычислено \%: $\mathrm{C}-$ $26,18, \mathrm{H}-3,27, \mathrm{~N}-35,64$, найдено, \%: $\mathrm{C}-25,91, \mathrm{H}-2,98, \mathrm{~N}-35,57$.

1. Rusinov V. L., Ulomskii E. N., Chupakhin O. N., Charushin V. N. Azolo[5,1-c]-1,2,4-triazines as a new class of antiviral compounds. Russ. Chem. Bull., Int. Ed. 2008, 57(5): 985-1014. DOI: $10.1007 / \mathrm{s} 11172-008-0130-8$.

2. Karpenko I., Deev S., Kiselev O., Charushin V., Rusinov V., Ulomsky E., Deeva E., Yanvarev D., Ivanov A., Smirnova O., Kochetkov S., Chupakhin O., Kukhanova M. Antiviral properties, metabolism, and pharmacokinetics of a novel azolo-1,2,4-triazine-derived 
inhibitor of influenza A and B virus replication. Antimicrobial Agents and Chemotherapy. 2010, 54(5): 2017-2022. DOI: 10.1128/AAC.01186-09.

3. Denicola A., Radi R. Peroxynitrite and drug-dependent toxicity. Toxicology. 2005, 208(2): 273-288. DOI: 10.1016/j.tox.2004.11.023.

4. Liu J., Dang Q., Wei Z., Zhang H., Bai X. Parallel solution-phase synthesis of a 2,6,8,9-tetrasubstituted purine library via a sulfur intermediate. J. of Combinatorial Chemistry. 2005, 7(4): 627-636. DOI: 10.1021/cc049819p

5. Rusinov V. L., Ulomskii E. N., Chupakhin O.N., Petrov A. Yu., Sharonov E. A. Nitroazines. 9. Characteristic features of nucleophilic substitution of the nitro group in dihydroazolo[5,1-c] [1,2,4]triazines. Chemistry of Heterocyclic Compounds. 1989, 25(2): 209-213. DOI: 10.1007/BF00479921. 